\title{
Movement Strategies in a Haptic Search Task
}

\author{
Vonne van Polanen*
}

Wouter M. Bergmann Tiest

Helmholtz Instituut, Universiteit Utrecht
Astrid M.L. Kappers

\begin{abstract}
Movement strategies were investigated in a haptic search task where participants indicated whether a target was present among a varying number of items. Hand movements were classified according to two criteria into three movement types. Results indicated that an easy search was performed with a parallel strategy, while in a more difficult search a serial movement strategy is used. Short, fluent movements were made in the easy search, whereas the difficult search showed more detailed movements and this amount increased when more items were to be searched. These differences between the search tasks remained when corrected for travelled distance, while path length could partly explain dissimilarities between trials with a target present and absent. Overall, these results indicate that movement strategies are adjusted primarily to the search condition and the salience of the target, and less to the presence of a target and set size.
\end{abstract}

KEYWORDS: Haptic search, pop-out, exploratory movements

INDEX TERMS: H.1.2 [Models and Principles]: User/Machine Systems-Human information processing; J.4 [Social and Behavioral Sciences]: Psychology

\section{INTRODUCTION}

In everyday life, we often perform haptic searches, for example, when trying to find our keys in our bag or a lightswitch in the dark. When searching for objects, some searches are easier than others. Certain features are more distinguishing from other features and have the property to 'stand out' between others and are therefore more easy and faster to find. This phenomenon is called the pop-out effect. In this case, the target property that is to be found pops out between distractor items that do not exhibit the target property [1]. Sometimes the pop-out effect can disappear when the properties of target and distractor are interchanged; this is known as a search asymmetry. For example, when searching for a rough item between less rough items, the search is more efficient than when target and distractors are reversed [2]. In other words: the rough item pops out between the smooth items, but a smooth item does not pop out between rough ones.

In the situations of the example above, often the distinction between a parallel and a serial search strategy is made. In the case of a difficult search, a serial search strategy is used and all items must be explored one by one to find out whether it has the target property. In a parallel search, multiple items can be searched at once, which makes the search much easier. In an experiment, typically reaction times are measured to illustrate the difference between these two search strategies. In most search tasks, the task is to find out whether a target is present between distractors and

* e-mail: v.vanpolanen@uu.nl

IEEE World Haptics Conference 2011

21-24 June, Istanbul, Turkey

978-1-4577-0297-6/11/\$26.00 @2011 IEEE the number of items to be searched is varied. Reaction times are higher in the serial strategy; it takes longer to find a target property. Furthermore, because additional time is needed for each extra item, the reaction time tends to increase with the number of distractors; the slope of reaction time against the number of items (the search slope) is steeper. The steeper slope indicates that the task becomes increasingly more difficult when the total number of items to be searched increases. In the case of a parallel strategy, the reaction time is independent of the number of items. Because items are searched in parallel, the reaction time is predicted to remain constant as the number of items to be searched increases, i.e. a flat search slope is expected. The task is not much more difficult when the total number of items increases, because the target object exhibits a property that is easy to recognize between other items that do not have that certain property. In other words: the target pops out. So, a pop-out effect is associated with a parallel search strategy and search times that depend on the number of items with a serial strategy.

However, the distinction between parallel and serial search strategies seems to be not as strict as suggested above. A range of search slopes has been found in visual search [3]. In haptic search, a slope of zero has rarely been found and the analysis of hand movements offers a better perspective for the distinction between serial and parallel search [2]. Plaisier et al. found that a single hand-sweep was enough to detect a rough target among smooth distractors, whereas more detailed movements were needed in search for a smooth target among rough distractors [2]. So it seems that the analysis of hand movements in active haptic search is important for the interpretation of search slopes. The type of exploration might be a better discriminator between a parallel and a serial strategy in haptic search than the absolute search slope value.

The notion that exploration varies with the target property of the object has been demonstrated by Lederman and Klatzky [4]. They investigated exploratory movements of various objects and classified a number of movements that were used consistently for determining a certain object property. For example, for exploring texture like roughness, a lateral motion is used. However, as stated above, Plaisier et al. showed that even these lateral movements can be subdivided into more categories, like a single sweep or a more detailed movement [2]. This indicates that the exploratory strategy can differ between different search conditions of the same property. They only investigated a few trials, so no further comparisons could be made between situations in which a target was present or absent, or the number of items. It is not unimaginable that even within a certain search task the exploration strategies could vary according to the circumstances.

For this study, we wanted to investigate the movement strategies that are used in different search conditions. Moreover, we examined if certain hand movements or hand movement types were used more often in different searches. Therefore, a classical search task similar to Plaisier et al. [2] was used in which participants had to find out whether a target was present among distractors. As target and distractors, movable and anchored stimuli were used in two conditions. In the movable-target condition, participants had to determine if a movable target was present among anchored distractors and in the anchored-target 
condition it was the other way around. The number of distractors was varied to indicate whether a parallel or serial search strategy was used in each condition.

These serial and parallel search tasks were further examined by identifying the proportion of movement types used in both search tasks. To determine the type of hand movements, we created an algorithm that classified each trial into a movement type category using only two variables. Movement strategies used to perform a search task might be determined by the participant beforehand or during the task. For example, participants know which property the target has and they can anticipate on this knowledge by adjusting their hand movements. In this way, different movement patterns might be found between the two conditions. However, participants do not know whether a target is present or how many items are to be searched and may adjust their movements while searching. So, the strategy used can be changed or adapted in time. This might lead to different strategies depending on targetabsent or present trials and/or number of items.

\section{METHODS}

\subsection{Participants}

Eleven participants took part in the experiment. Due to a large number of errors in one condition (more than $10 \%$ of the total number of trials in a condition), one participant was excluded from the analysis. The remaining 10 participants ( 3 males, 7 females) had a mean age of $21 \pm 3$ years and were all right-handed according to Coren's test [5]. They used their dominant hand to perform the experiment. Participants gave informed consent and were paid for their participation.

\subsection{Apparatus}

The stimulus set consisted of square laminated chipboard displays of 20 by $20 \mathrm{~cm}$ in which holes had been drilled to fit $3,5,7,9$ or 11 items. The holes were located randomly on the display and items on the display were at least $1 \mathrm{~cm}$ apart and at least $2 \mathrm{~cm}$ from the edge of the display, at the same locations as used in [2]. There were two displays for each possible number of items. The items on the display consisted of ball transfer units in which the ball could either move or was anchored (Figure 1A). The anchored ball transfer units were produced by gluing the ball to its casing. Except for their ability to move, the items were indistinguishable. The diameter of a ball transfer unit was $31 \mathrm{~mm}$, with a ball of $15 \mathrm{~mm}$ in diameter. An example of a filled display is illustrated in Figure 1B.

The displays were presented onto a table, to the left of a handrest. The handrest was leveled with the displays, so participants could easily slide from the rest to the display. A metal strip (15 mm wide) was fixed between the handrest and the stimulus display, to trigger the start of the measurement. When participants moved over the strip, a change in potential started the clock. The end of the measurement was induced by a verbal response from the participant, recorded with a microphone placed in front of the participants. The time difference between touching the strip and the response was the reaction time.

Hand movements were recorded using an NDI Optotrak Certus system. Two infra-red emitting diodes (IRED) were placed on the right, dominant hand of participants: one on the nail of the index finger and one on the back of the hand near the wrist. Data were sampled with a frequency of $100 \mathrm{~Hz}$, resulting in $10 \mathrm{~ms}$ accuracy for reaction times and hand movement data. The spatial accuracy of the Optotrak was $0.2 \mathrm{~mm}$.

\subsection{Task}

The experiment consisted of two conditions. In the movable-target condition all items were anchored if no target was present (target-
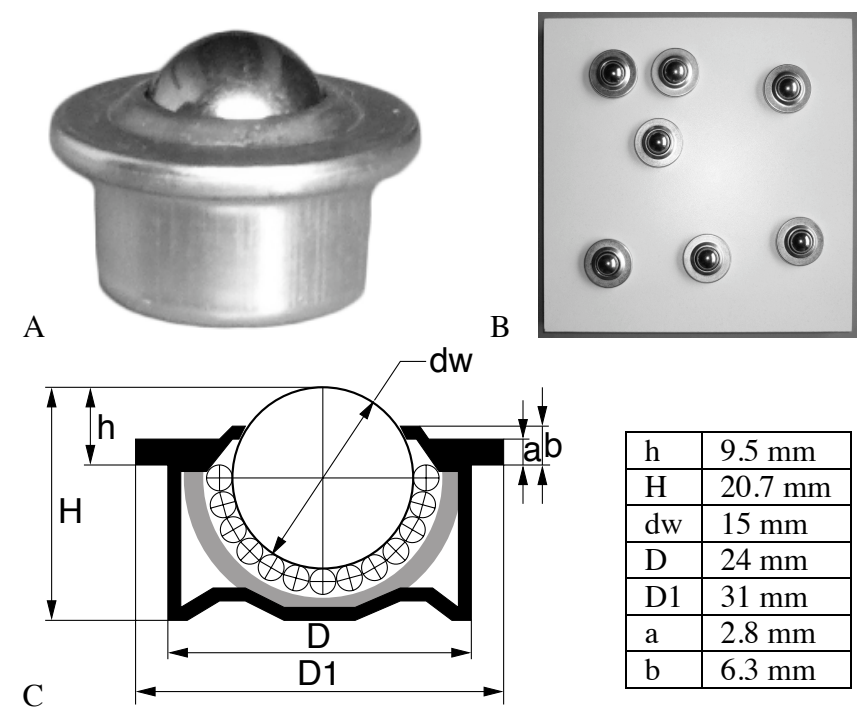

Figure 1. A: a ball transfer unit. B: example of a stimulus display with 7 items. C: cross-section of a ball transfer unit and list of dimensions.

absent trials), or one item was movable (target-present trials). In the anchored-target condition all items were movable if no target was present (target-absent trials), or one item was anchored (target-present trials).

Participants moved their hand over the display and had to respond as quickly as possible whether a target was present or not by calling out the Dutch equivalents of 'yes' and 'no'. They were instructed to determine as fast as possible whether a target was present or not, but also to make as few mistakes as possible. They were told that they could move their hand over the display in the way they preferred.

\subsection{Procedure}

First, the task and procedure were explained to the participants. Next, they put on the blindfold and were seated in front of the display. Participants wore hearing protectors and listened to white noise during the trials to block out the rolling noise and subsequent clues from the ball transfer units.

Before each trial, participants placed their hand on the handrest next to the display with their index finger on a start position, indicated by a small bump. The white noise was started to indicate that they could start their movement. Then, they moved their hand over the display, starting on the right-hand side. After they had responded verbally, the white noise was turned off and they received feedback about their answer. Incorrect trials were repeated at the end of a session.

The order of the two search conditions was counterbalanced between participants. Each condition was divided into two sessions of approximately 45-60 minutes. A session was preceded by a training session. At the first session of a condition, participants were told what to search for and 15 practice trials were presented in order to get comfortable with the nature of the task. During practice, the participants were encouraged to try out different strategies and to find a strategy in which they could perform the task as fast and as accurately as possible. If necessary, more practice trials were presented until 9 out of 10 trials were answered correctly before the actual experiment began. For the second session of a condition the same procedure was followed, except that a minimum of 10 practice trials were carried out until 9 out of 10 trials were correct. 
The location of the target was randomized among trials and the displays were rotated in the experiment to obtain as many item locations as possible. In a condition a target was never presented twice at the same location. The number of items and the target position were varied randomly. For each number of items $(3,5$, ...11), 28 trials were presented, resulting in a total of 280 trials. In half of the trials a target was present and in the other half the target was absent. The trials in a condition were divided over the two sessions in a way that each number of items was presented equally often in a session (i.e. 14 trials per number of items of which half contained a target in one session).

\subsection{Analysis}

A total of $106(4 \%)$ incorrect answers were given and those trials were repeated. Only correct trials were analyzed. Due to measurement errors 6 trials $(0.2 \%)$ were excluded from the reaction time analysis and 67 trials $(2 \%)$ from the Optotrak analysis. The measurement errors for the Optotrak mainly involved a too large amount of missing data points, because the marker was not visible to the camera.

\subsubsection{Reaction time analysis}

Mean reaction times were determined for each number of items in each condition. Values that differed more than three standard deviations from the mean were removed. Each value was plotted against the number of items for target-present and target-absent trials separately and a linear regression line was fitted through the data to determine the relation between reaction time and set size (i.e. the search slope). The slope and intercept of each regression line were calculated.

\subsubsection{Optotrak analysis}

Missing values $(<1 \%)$ in the hand movement data were interpolated using a second order polynomial function. Then, a moving average filter with a length of ten points $(0.1 \mathrm{~s})$ was used for smoothing the movement tracks. Pilot data showed that the marker on the index finger usually crossed the metal strip first, so the start of a hand movement measurement was determined at the point the index finger passed the middle of the metal strip. The end of the hand movement was determined as many data points later as the reaction time for that trial, which corresponded to the time the participants gave a verbal response. Participants mainly moved their whole hand and did not spread their fingers, so the movement track of the finger marker was sufficient to describe the whole hand movement. Only data from this marker were used in the further analysis.

\subsubsection{Classification algorithm}

After inspecting the data, it seemed that movements could be categorised roughly according to the fluency and covered area across the display. Two variables were used to distinguish between the various movement types in the dataset. These were vertical $(\mathrm{y}-)$ range and the number of turning points. The y-range was calculated by subtracting the lowest $y$-coordinate from the
Table 1. Decision tree used for classification

\begin{tabular}{|c|c|c|c|}
\hline & \multicolumn{2}{|c|}{ y-range $(\mathrm{mm})$} \\
\hline & & $\leq 80$ & $>80$ \\
\hline \multirow{2}{*}{ 象 } & $\leq 14$ & sweep & circle \\
\hline & $>14$ & \multicolumn{2}{|c|}{ scribble } \\
\hline
\end{tabular}

highest $\mathrm{y}$-coordinate. The turning points were computed for $\mathrm{x}$ - and $y$-direction separately. Movements could be divided in horizontal (i.e. left or right; x-direction) and vertical (i.e. up or down; ydirection) components. The total number of turning points was calculated by summing the changes in movement direction for horizontal and vertical movement components. Trials were classified into a certain movement type according to the values of the two variables, as indicated in Table 1. Based on this classification, the proportion of each movement type was calculated for all conditions and number of items. Criterion values were set at $80 \mathrm{~mm}$ for $\mathrm{y}$-range and 14 for the number of turning points, which matched the subjective classification of the first author. Qualitatively, the movements can be described as follows: a scribble is a detailed movement over the display, with many changes in direction. A circle is a movement with a vertical component. It can be just an up- or downward movement, or roughly representing one or more circle movements. The sweep is one or multiple more or less straight, horizontal movement(s) over the display. Examples of each movement type are illustrated in Figure 2. Note that the track of the finger marker is shown, but that the perceptive area of the hand extends up to about $15 \mathrm{~cm}$ below this track.

\subsubsection{Statistics}

Each of the three movement types were analysed separately with a 2 (condition) $\times 2$ (target presence) Analysis of Variance (ANOVA). Significance level was set to 0.05 and post-hoc tests were performed using paired sampled t-tests with a Bonferroni correction. Only interesting comparisons were made in the posthoc tests (e.g. differences in condition or target presence were examined, but not combinations of those factors). Data for the ANOVAs were averaged over the number of items to simplify the interpretation, but regression analysis was carried out on each movement type for each condition and target-absent and present trials separately to determine the change in movement types with number of items. Only significant results will be presented.

\section{Results}

\subsection{Search slopes}

The change of the reaction times as a function of the number of items confirmed a pop-out effect for the movable-target condition. In this condition, an intercept of $0.85 \mathrm{~s}$ and a search slope of only

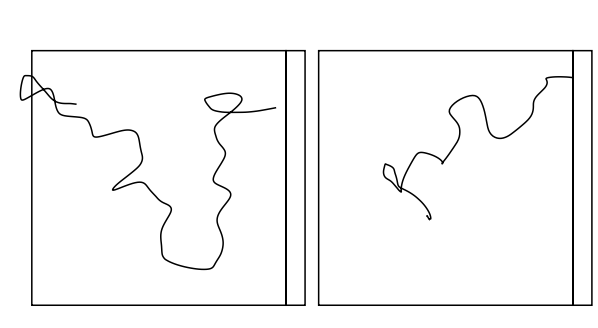

Scribble

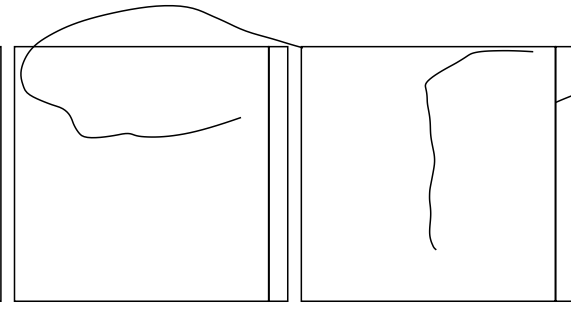

Circle

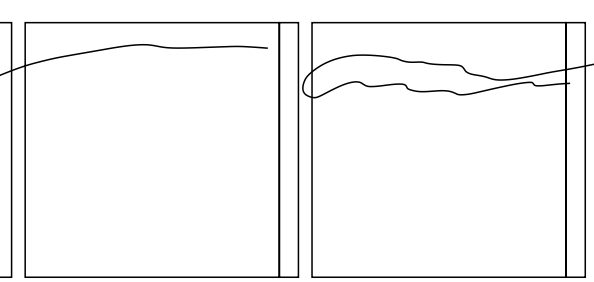

Sweep

Figure 2. Examples of movement types. 
$39 \mathrm{~ms}$ per item were found for target-present trials. This means that the reaction time is independent of the number of items to be searched. When the target was anchored, the intercept was $1.25 \mathrm{~s}$ and the slope was $105 \mathrm{~ms}$ in target-present trials, indicating an increase of reaction time with the number of items. It seems that the conditions differed in search strategy. Based on these results, we expected a more serial search strategy in the anchored-target condition and a more parallel strategy in the movable-target condition.

\subsection{Hand movement types}

The percentage of movement type in each condition is plotted in Figure 3. Statistical effects will be described for each movement type separately. The results of the regression analyses are shown in Table 2.

When looking at Figure 3, clear differences between the conditions are seen. In the target-present trials it seems that sweeps are most common. This is more apparent in the movabletarget condition. In contrast, in the target-absent trials the proportion of sweeps is decreased. In the anchored-target condition, the scribble is the dominant movement type, while in the movable-target condition this place is reserved for the circles. Statistical differences between conditions and target-absent and present trials are further discussed for each movement type separately.

\subsubsection{Scribble}

There were main effects of condition and target presence on the proportion of scribbles $\left(F_{1,9}=40, p<0.001 ; F_{1,9}=28, p<0.001\right.$, respectively). The effect of condition showed that there were more scribbles in the anchored-target condition. In addition, there were more scribbles in the target-absent trials. The condition $\times$ item interaction $\left(F_{19}=15, p=0.004\right)$ revealed that in the anchored-target condition the change in scribble proportion from target-present to target-absent trials was much larger than when searching for a movable target. Regression analysis showed an increase of scribbles with the number of items for both target-present $(r=3.91$ $\% /$ item, $p=0.001)$ and absent trials $(r=4.88 \% /$ item, $p=0.02)$ in the anchored-target condition and absent trials only in the movabletarget condition ( $r=2.07 \% /$ item, $p=0.02)$.

\subsubsection{Circle}

More circle movements were made in the movable-target condition than in the anchored-target condition (condition effect, $\left.F_{1,9}=5.5, p=0.04\right)$. The effect of target presence $\left(F_{1,9}=16, p=0.003\right)$ showed that more circles were used when no target was present. However, the interaction between condition and target presence $\left(F_{1,9}=39, p<0.001\right)$ revealed that this difference was only seen in the movable-target condition. Also the difference between the two search tasks only held for target-absent trials. Regression analysis indicated a decrease in circles with the number of items in the search for an anchored target when no target was present $(r=-3.09$ $\% /$ item, $p=0.03$ ).

\subsubsection{Sweep}

More sweeps were made in the movable-target condition $\left(F_{1,9}=9.9, p=0.01\right)$. Furthermore, more sweeps were made when a target was present than when it was absent $\left(F_{1,9}=55, p<0.001\right)$. The number of sweeps decreased with the number of items in the anchored-target condition for both target-present $(r=-3.23$ $\% /$ item, $p=0.005)$ and absent trials $(r=-1.80 \% /$ item, $p<0.05)$.

\subsubsection{Correction for distance}

Although the $y$-range is relatively independent of travelled distance, the number of turning points will increase with distance, because one always has to move back and forth across the display.
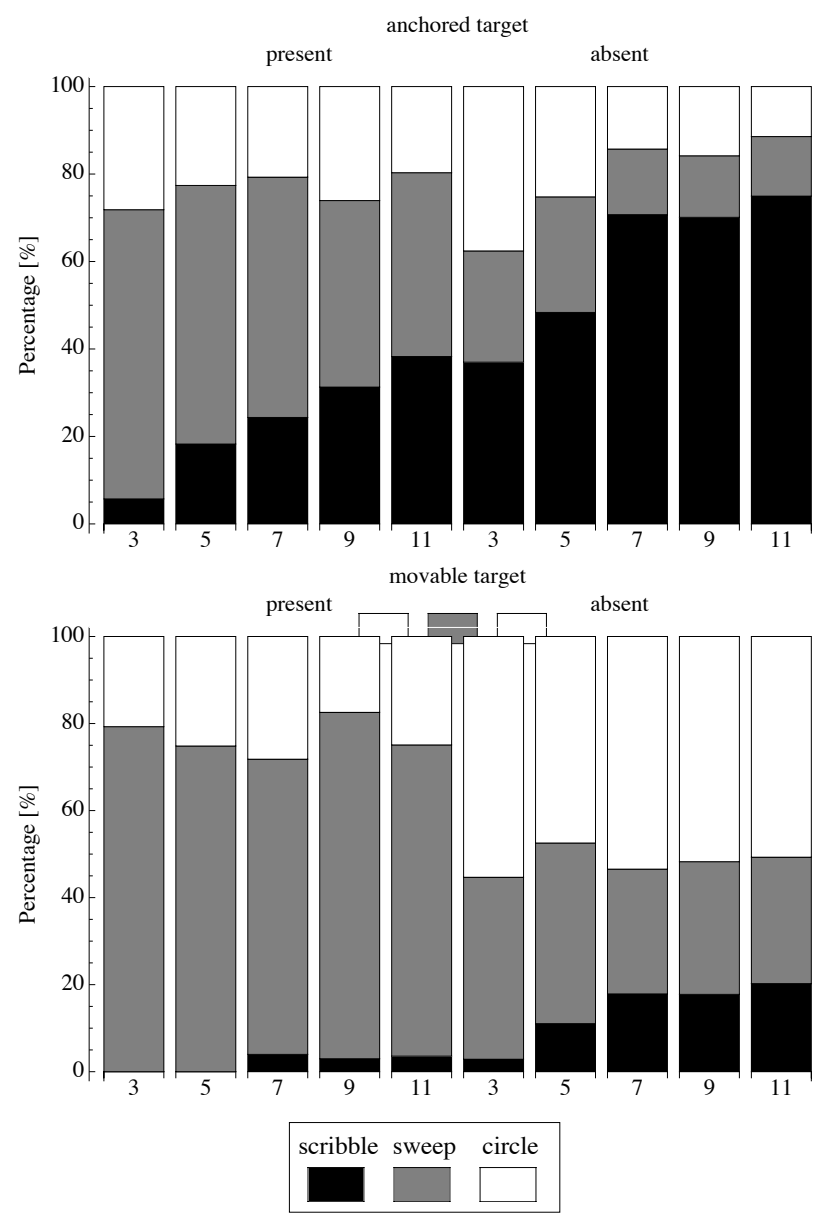

Figure 3. Proportion of movement type used per condition in percentages. Top: anchored-target condition. Bottom: movabletarget condition. The left five bars represent target-present trials and the right five bars target-absent trials for each of the five numbers of items.

Table 2. Slopes of the regression analysis for the anchored-target condition (left) and movable-target condition (right) in \% per item. Values are given for each movement type, for target-present (upper value) and absent trials (lower value) separately. *significant at 0.05 level, ** significant at 0.01 level.

anchored movable

\begin{tabular}{ccc}
\hline scribble & $3.91^{* *}$ & 0.51 \\
& $4.88^{*}$ & $2.07^{*}$ \\
\hline circle & -0.67 & 0.03 \\
& $-3.09^{*}$ & -0.25 \\
\hline sweep & $-3.23^{* *}$ & -0.54 \\
& $-1.80^{*}$ & -1.83
\end{tabular}

Table 3. Average relations between different conditions with respect to travelled distance. Ratios displayed are the lowest mean value divided by the highest mean value.

\begin{tabular}{lll} 
& Target & Distance ratio \\
\hline \multirow{2}{*}{ Movable vs anchored } & Present & 0.94 \\
& Absent & 0.79 \\
\hline \multirow{2}{*}{ Present vs absent } & Movable & 0.54 \\
& Anchored & 0.45 \\
\hline
\end{tabular}


Possibly the differences in movement types found between the conditions could be explained by a deviation in movement duration. Since two trials can differ in speed, it is more appropriate to correct for the amount of travelled distance instead of reaction time. For example, two identical movements with the same number of turning points performed at an unequal speed differ in the number of turning points per second, but not per $\mathrm{cm}$.

We wondered whether the differences found above still existed when corrected for distance. The movable-target condition was compared with the anchored-target condition (for target-present and absent trials separately) and target-present trials were compared with target-absent trials (for the movable-target and anchored-target condition separately). The average distance relations between the conditions are shown in Table 3 . These ratios were obtained by dividing the mean travelled distance of one condition by the other. To correct for distance, we compared the conditions with aid of the ratios in Table 3. For instance, if we want to investigate the movable condition and the anchored condition when a target was present, we compared $100 \%$ of the movable condition with the first $94 \%$ of each trial in the anchored condition.

Results are shown in Figure 4. Still large differences between the two search tasks were found when correcting for distance. When comparing target-present and absent trials, the difference in scribbles disappeared in the movable-target condition, but there were still a little more circles in the target-absent trials. In the anchored-target condition the scribble dissimilarities were reversed, with more scribbling movements in the target-present trials. Nevertheless, this analysis should be interpreted with care, since it was only corrected for average distance ratios but the movement strategies used varied within a condition and even within participants.

\section{Discussion}

In this study it was investigated which movement strategies are used in different conditions when performing a search task. The task was to indicate whether a target was present or absent in the search for an anchored target among movable distractors or a movable target among anchored ones. Reaction times between entering the search display and a verbal response and hand movements were measured during the experiment. Furthermore, based on two criteria three movement types were defined and each trial was classified into one of the movement types. The movement types consisted of fluent movements (sweep, circle), and non-fluent movements, indicative of a detailed search (scribble). Overall, it was seen that sweeps and circles were dominant movement types, especially in the movable-target condition, while scribbles were more dominant in the search for an anchored target.

Changes in reaction time with the number of items (search slopes) indicated that the search for an anchored target was less efficient and the target did not pop out between the distractors. On the other hand, in the search for a movable target the reaction time was independent of the number of items; a flat search slope was found. These results show that the search for an anchored target is a more difficult search than the search for a movable target and therefore there might also be a difference in exploration strategy.

Analysis of hand movements confirmed the distinction between a serial and a parallel strategy between the two search tasks. In the condition with a movable target, an easier search task, the main movement types were sweeps and circles. These are fluent movements and the hand goes over the display just once or twice. The hand moves in a more or less straight line or a circle and several items are felt at once. The items that touch the fingers and the palm of the hand can be judged to contain a target or not in a single movement and this indicates a parallel search. An
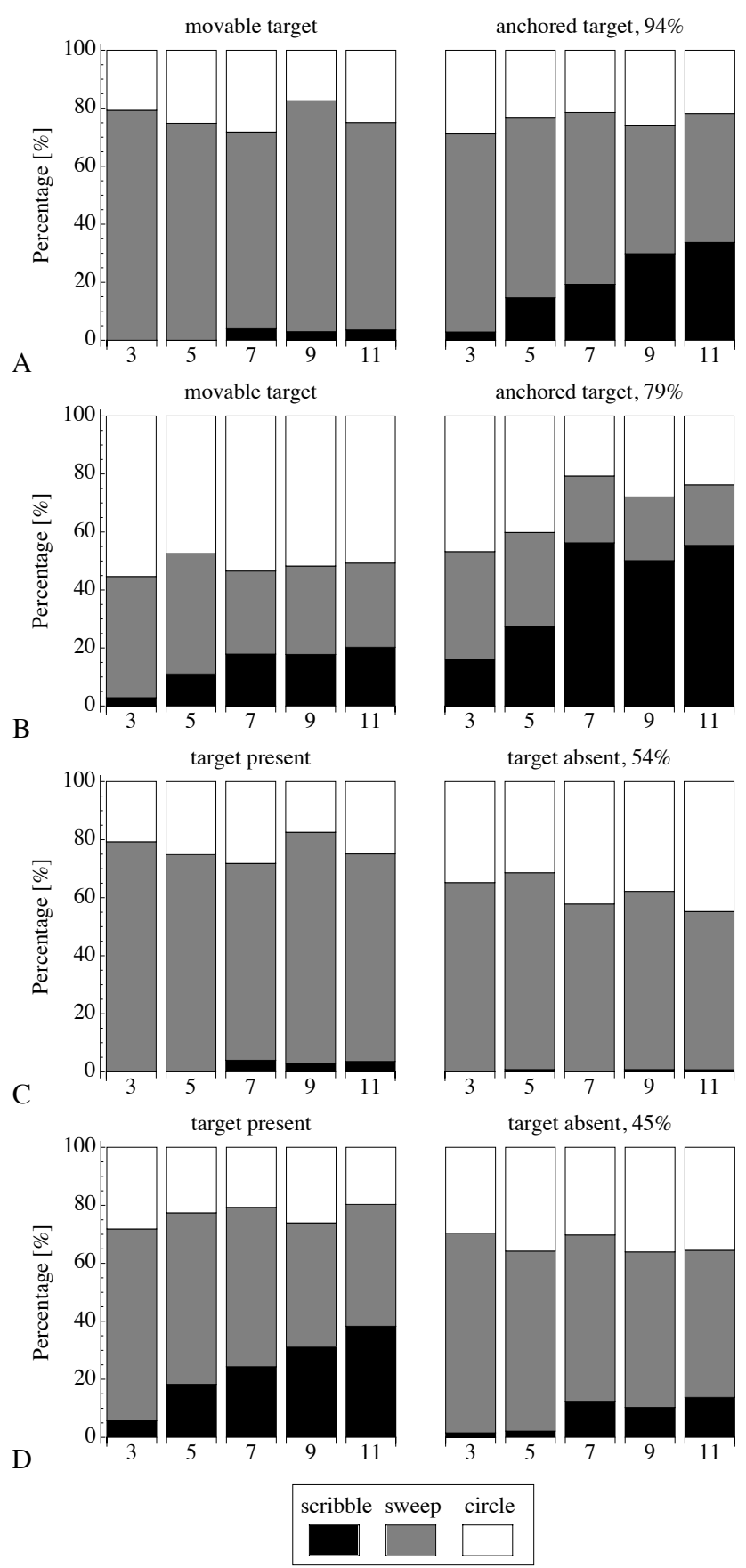

Figure 4. Comparison between search conditions (A: target present, $\mathrm{B}$ : target absent) and between target-present and absent trials (C: movable target, D: anchored target) corrected for distance. Bars are displayed for each number of items.

interesting finding was that there were barely any changes with the number of items in the movable-target condition, except for a small increase in scribbles in the target-absent trials. This supports the notion of a parallel search, which is independent of the number of items. Since a lot of items are felt at the same time, it does not matter how many items are to be searched, because it will take the same movement.

In contrast, in the more difficult search, i.e. the search for an anchored target among movable distractors, more scribbling movements were seen. This was especially the case when more items were to be searched and no target was present. Thus, less 
fluent movements are made, because a detailed search is necessary in this condition. The items need to be searched one by one to determine whether a target is present and with more items on the display, more items need to be searched resulting in a longer and more intensive search.

The differences in movement strategies between a difficult and easy search are consistent with the results of a similar experiment by Plaisier et al. [2]. They found a pop-out effect for the search for a rough item among smooth distractors. Hand movement analysis indicated that participants used a hand sweep to find out whether a target was present. In the reversed situation, i.e. a smooth target among rough distractors, the search was more difficult and a detailed search was necessary. So it seems that the search strategies are not directly related to the stimulus feature, but more to the saliency or distinctiveness of the target. If a target pops out, it is believed to be more salient and participants seem to modify their movement strategy towards this. In fact, in this study the differences in movement types remained present even when correcting for travelled distance.

In contrast to these results, Smith, Gosselin and Houde found no differences in search strategy for a raised square and an inverted square, although the former was found to be more salient [6]. In both conditions participants used a side-to-side movement to find the target. Still, participants did adjust the contact force with the surface according to the two tasks. An explanation might be that in their study only the index finger could be used for exploration and therefore no parallel strategy was possible. In fact, the exact movement strategy used seems to depend heavily on task circumstances and instructions. The current experiment investigated a two-dimensional stimulus display, but different movement strategies might be expected for three-dimensional objects. Lederman and Klatzky found distinctive movements for the exploration of various object properties [4]. When for instance determining 3D-shape, enclosure of the object is used, whereas lateral motion is used for texture exploration. In any case, the present study shows that although the same general motion is used in the two search conditions, this can be qualitatively subdivided into more distinctive movement strategies.

Apart from the differences between the two search conditions, participants also adjusted their movement strategy to the presence or absence of a target. Note that they could not determine this beforehand, because they did not know whether a target was present. The movement strategy therefore had to be changed in time, during the search task. In the case when a target was absent, less sweeps were made. Informally it was observed that the remaining sweeps were often rather long horizontal movements, which is not surprising. It seems that when no target was present, even though participants could have felt all the items by moving once over the display, they tended to move over the display again to be certain they did not miss anything. Moreover, the differences between the two search tasks were amplified in the target-absent trials, with a preference for scribbles in the anchored-target condition, whereas circles were favoured in the search for a movable target. However, when correcting for travelled distance in the movable-target condition, it seemed that searching in targetabsent trials might be more a matter of continuation of the movements in the target-present trials. This is expected, since participants did not know whether a target was present. Yet, some differences remained. More vertical movements were made when no movable target was present. Furthermore, in the anchoredtarget condition relatively more scribbles were made in the when a target was present. A possible explanation for this could be that participants often rubbed the target, to make sure it was indeed anchored.

The changes in movement strategy with number of items appear to occur mainly in the search for an anchored target. Because this target was searched in a serial way, the task was more difficult when more items needed to be searched and this resulted in a change in movement strategy. With more items a longer and intensive search was needed. This was reflected by a decrease in the amount of fluent movements, possibly in favour of more scribbling movements, which increased. Although not analysed in this study, these changes with item number might also be partly explained by an increase in travelled distance. In contrast, like stated above, because of a parallel search the hand movement types were independent of the number of items when searching for a movable target. As a result, there were barely any changes with number of items for this condition.

In conclusion, there are many differences in exploration movements in an easy and difficult search. An easy search results in a parallel strategy and a difficult search is performed with a serial strategy. In an easy search the hand movements are more fluent and barely any adjustments are made in response to more items. Conversely, in a difficult search less fluent and more detailed movements are made. In addition, the amount of fluent movements even decreased and the search is more thorough when more items need to be searched. Dissimilarities induced by target presence could be partly explained by a longer travelled distance in the case of an absent target. Overall, these results indicate that movement strategies are adjusted primarily to the search condition and the salience of the target and less to the presence of a target and set size.

\section{ACKNOWLEDGEMENTS}

This work was supported by the European Commission with the Collaborative Project no. 248587, "THE Hand Embodied", within the FP7-ICT-2009-4-2-1 program "Cognitive Systems and Robotics". The authors would like to thank Pieter Schiphorst for technical support.

\section{References}

[1] A. M. Treisman and G. Gelade. Feature-integration theory of attention. Cognitive Psychology, 12:97-136, 1980.

[2] M. A. Plaisier, W. M. Bergmann Tiest and A. M. L. Kappers. Haptic pop-out in a hand sweep. Acta Psychologica, 128:368-377, 2008.

[3] J. M. Wolfe. What can 1 million trials tell us about visual search? Psychological Science, 9:33-39, 1998.

[4] S. J. Lederman and R. L. Klatzky. Hand movements - a window into haptic object recognition. Cognitive Psychology, 19:342-368, 1987.

[5] S. Coren. The Left-Hander Syndrome: The Causes and Consequences of Left-Handedness, Vintage Books, New York, 1993.

[6] A. M. Smith, G. V. Gosselin and B. Houde. Deployment of fingertip forces in tactile exploration. Experimental Brain Research, 147:209218,2002 . 